\title{
Breast Reconstruction with Perforator Flaps in Poland Syndrome: Report of a Two-Stage Strategy and Literature Review
}

\author{
Boyang Xu Tong Liu Chunjun Liu \\ Department of Aesthetic and Reconstructive Breast Surgery, Plastic Surgery Hospital, Chinese Academy of \\ Medical Sciences, Peking Union Medical College, Beijing, China
}

\section{Established Facts}

- The latissimus dorsi muscle with implants is routinely used for Poland syndrome breast reconstruction but can yield unsatisfying results due to implant-related complications.

- Perforator flaps have mainly been used in revision surgery due to severe implant-related complications but are seldom discussed in the context of primary breast reconstruction in Poland syndrome.

\section{Novel Insights}

- Perforator flaps provide a reliable alternative for breast and chest-wall reconstruction in Poland syndrome.

- A 2-stage approach consisting of tissue expansion and deep inferior epigastric perforator flap transfer, successively, attained esthetic restoration of the breast, with satisfying breast symmetry and an inconspicuous scar.

\section{Keywords}

Poland syndrome $\cdot$ Breast reconstruction $\cdot$ Perforator flap

\begin{abstract}
Background: Poland syndrome is a congenital deformity presenting unilateral hypoplasia of the breast, pectoralis muscle, and ipsilateral skeletal anomalies. In complex female cases, the significant amount of soft tissue needed for reconstruction continues to be a surgical challenge. Perforator flaps offer alternative methods with sufficient tissue volume, minimal donor site morbidity, and natural cosmetic outcome; however, their role in Poland syndrome breast reconstruction is seldom discussed. This study aims to present a new 2-stage strategy for esthetic restoration of the Poland syndrome chest anomaly that achieves breast symmetry
\end{abstract}

while maintaining a scarless appearance. Case Presentation: A 38-year-old female presented with Poland syndrome on the right side, and amastia, athelia, and absence of pectoralis major muscle were observed. We present an innovative 2-stage approach consisting of tissue expansion and, successively, a deep inferior epigastric perforator flap transfer. A pleasing breast appearance had been achieved at 6 months of follow-up. A literature review regarding the use of free perforator flaps in Poland syndrome was conducted to demonstrate the applicability of this method. Seven articles described a total of 15 cases using free perforator flaps met the inclusion criteria and were summarized. A satisfactory correction was reported in all the cases. Conclusion: Perforator flaps provide reliable alternatives for Poland syndrome breast and chest-wall reconstruction. Proper patient selection and thorough assessment are vital to the success of the surgery. 


\section{Introduction}

Poland syndrome is a congenital deformity first introduced by Sir Alfred Poland in 1841 [1], and comprises defects of the pectoralis major muscle, an absence of the anterior axillary fold, aplasia or deformity of the ribs, and ipsilateral symbrachydactyly. In female patients, hypoplasia/aplasia of the breast and nipple-areolar complex is the primary reason for seeking reconstructive surgery [2]. Given the fact that the breast and chest-wall reconstruction in Poland syndrome is for esthetic reasons only, factors such as surgical complications, functional sequelae, and patient preferences should be taken into consideration when selecting reconstructive options.

Several techniques have been proposed to achieve the reconstructive goal for breast and chest-wall deformities in Poland syndrome. Mammary prosthesis and customized chest-wall implants have been used routinely but can yield unsatisfying results due to implant-related complications including capsular contracture, contour irregularities, migration, or discomfort [3-5]. The latissimus dorsi (LD) muscle is well deployed as autologous tissue [6]; however, to make up for the limited amount of tissue amount an LD flap provides, combining it with the use of a silicon prosthesis is hard to avoid, given the breast mound to be reconstructed and this compromises the advantages of autologous reconstruction [3]. The transverse rectus abdominal muscle (TRAM) flap offers a source of autologous tissue with sufficient quantity, but donor-site morbidity such as hernia/bulge formation and decreased abdominal wall strength restrict its use, especially in active patients [7]. Adipose injection, or lipomodeling, promises the least donor-site morbidity and a softness to the touch, but disadvantages such as multiple treatment sessions, a lack of donor-site adipose tissue, and fat necrosis as a potential complication could be problematic when using these techniques $[8,9]$. Other methods such as the laparoscopically harvested omental flap [10] have also been successful to a certain degree, but all have limits and associated complications.

Perforator flap is an alternative for autologous reconstruction while minimizing the damage of muscle function, which has made it a popular technique for breast reconstruction in recent decades [11]. There have been several reports on different flap donor sites for breast and chest-wall reconstruction of Poland syndrome, including deep inferior epigastric artery perforator (DIEP) [12], superficial inferior epigastric artery (SIEA) [13], superior gluteal artery perforator (SGAP) [14], and inferior gluteal artery perforator (IGAP) flaps [15]. However, as these were mostly independent case reports, the efficacy and reliability of this method has not been reviewed yet.

In this article, we present a clinical case of a female patient with Poland syndrome, in whom breast reconstruc-

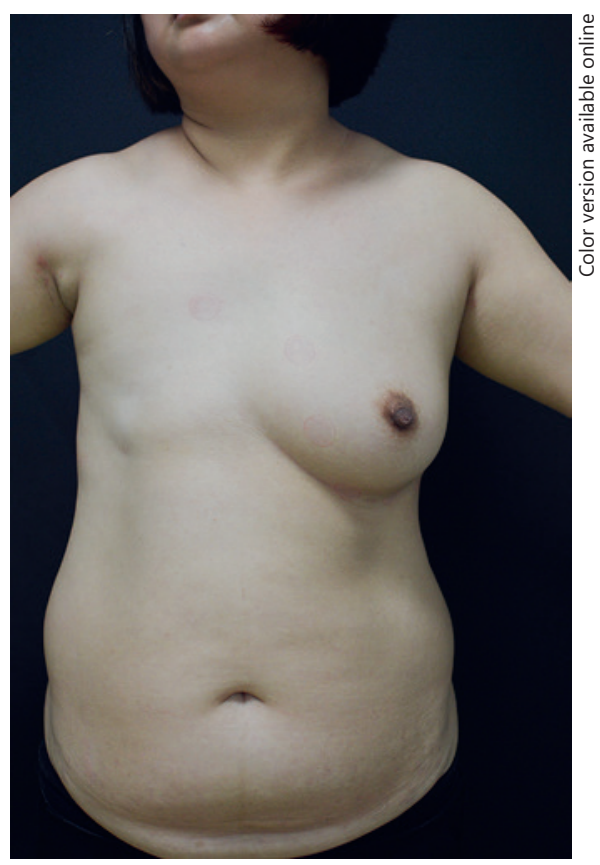

Fig. 1. A 38-year-old female patient with Poland syndrome, exhibiting unilateral breast hypoplasia, nipple-areolar complex absence, and chest-wall deformity.

tion was performed with an innovative 2-stage approach, consisting of tissue expansion and DIEP flap transfer, successively, to acquire esthetic restoration of the chest contour as well as breast symmetry. To demonstrate the applicability of this method, we also conducted a review of the literature regarding the application of free perforator flaps in Poland syndrome breast reconstruction.

\section{Case Presentation}

A healthy 38-year-old female presented with amastia, athelia, and absence of the pectoralis major muscle on the right (Fig. 1). A difference between her bilateral breast profile had existed since childhood and was aggravated after adolescence. Computed tomography and 3 -dimensional (3D) reconstruction of the chest revealed a mild rotation of the sternum and narrowing of the intercostal spaces, while the clavicular head of the pectoralis major muscle was intact (Fig. 2). A $3 \mathrm{D}$ surface scanning reconstruction was carried out to estimate the bilateral discrepancy in soft-tissue volume in the chest wall as well as in the overlying breast mounds, which was approximately $630 \mathrm{~mL}$. The method is described in one of our previous studies [16].

A thorough explanation to the patient regarding all alternative surgical options and possible complications was conducted by the senior surgeon (C.L.), after which the patient expressed strong rejection of silicone prosthesis and any adjustment of the contralateral breast. As a result, the autologous DIEP flap was selected, utilizing the excessive skin and adipose tissue in her lower abdomen.

The patient did not want to visible scars, so tissue expansion was justified to expand the constricted skin on the affected side. A round, $400-\mathrm{mL}$ tissue expander was placed in the subcutaneous plane through an endoscopic axillary approach as the first stage of the procedure. Over 5 months, the expander was inflated to 600 


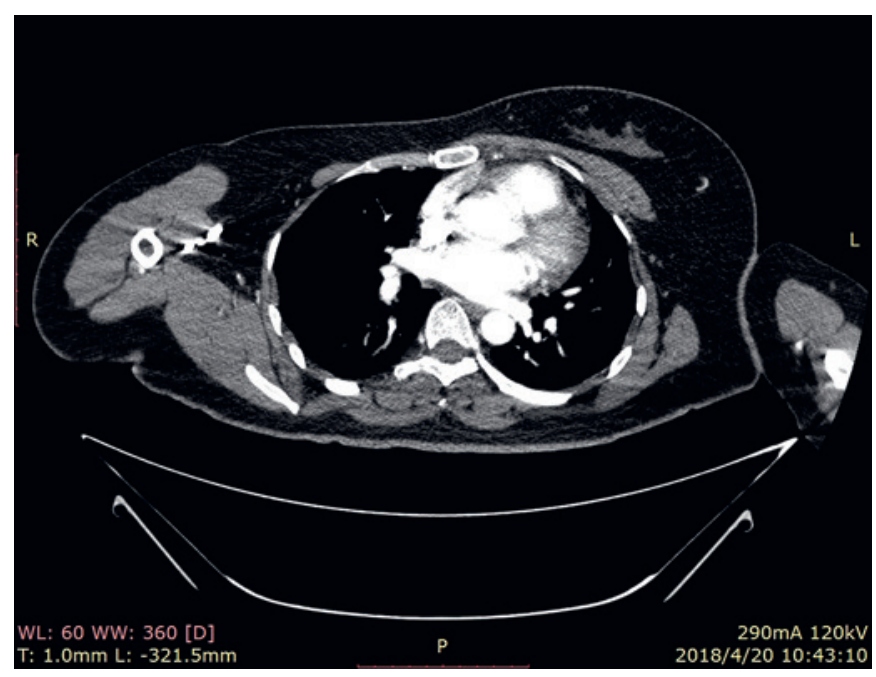

Fig. 2. Computed tomography revealing a mild rotation of the sternum. The pectoralis major muscle was absent but the clavicular head and other pectoral and chest muscles were intact.

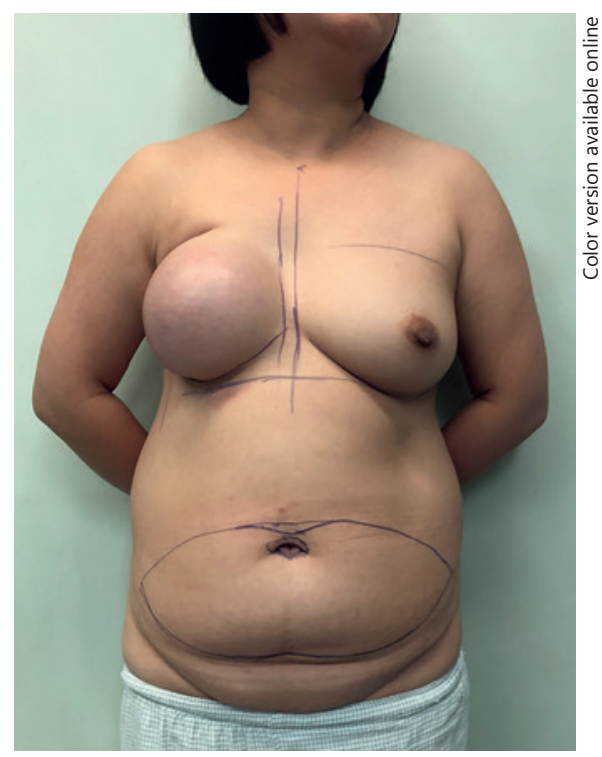

Fig. 3. Preoperative appearance after 5 months of tissue expansion.

$\mathrm{mL}$ to address the difference between bilateral breast volume and chest-wall contour (Fig. 3).

CT imaging was performed to evaluate the recipient and donor vessels. Information was collected, including the location and intramuscular course of the deep inferior epigastric vessels from their origin and the caliber of the perforators, and the dominant perforators were identified. The internal mammary and thoracodorsal vessels of the affected side were confirmed to be normal. Considering access through the prior lateral thoracic incision, and to avoid unsightly scarring near the torso midline, the thoracodorsal vessels were chosen as the first candidate recipient vessels.

Preoperatively, the upper limit of the unaffected breast and midsternal line were marked with the patient in a standing position. The inframammary fold (IMF) of the to-be-reconstructed right breast was designed $1 \mathrm{~cm}$ higher than the left side, considering the anticipated lowering of the IMF as a result of the abdomi-

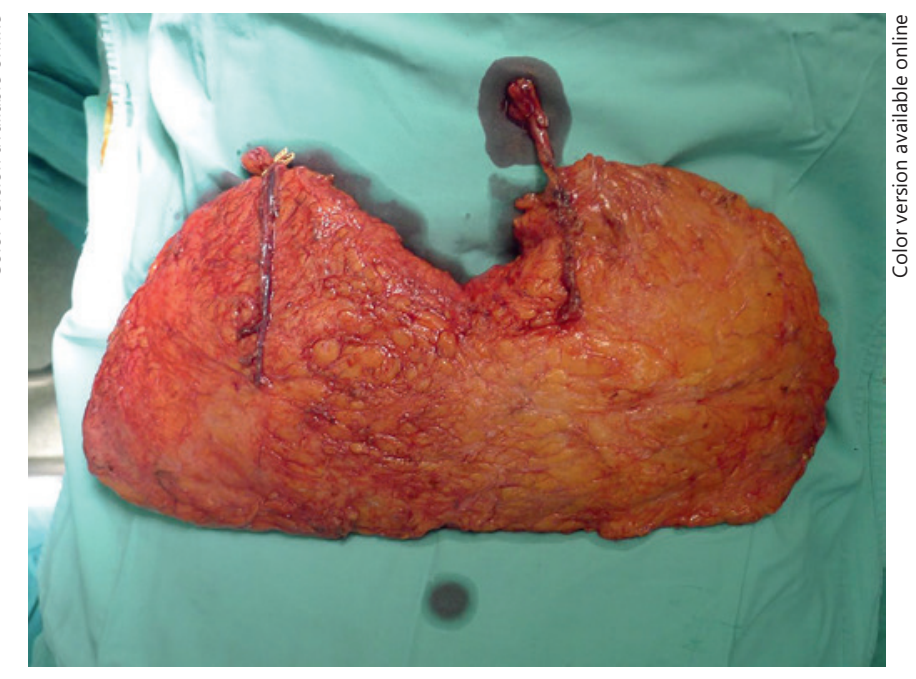

Fig. 4. DIEP flap harvested with bilateral pedicle vessels.

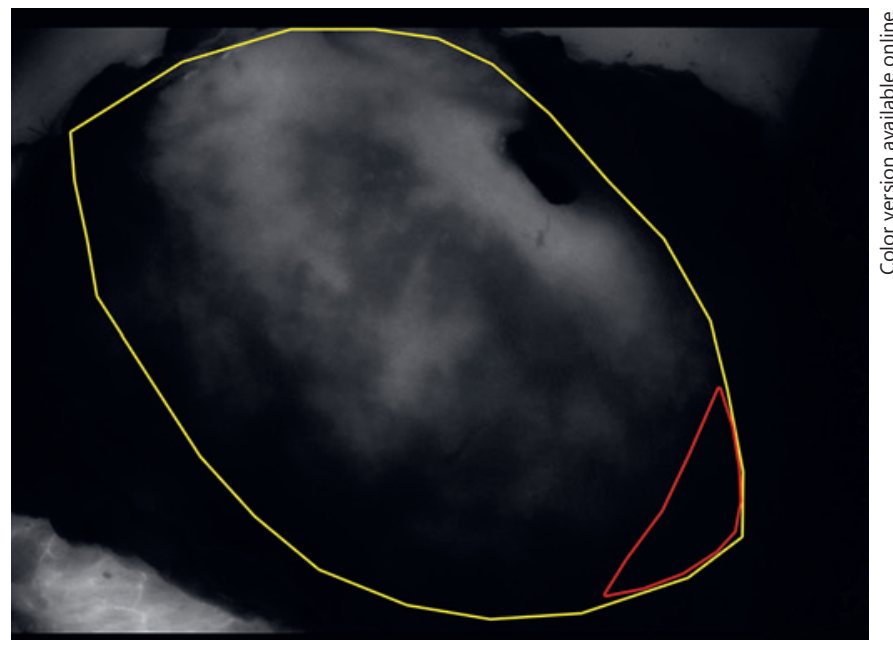

Fig. 5. Indocyanine green imaging evaluated the perfusion of the flap (the yellow line), showing a relatively weak fluorescence signal in the distal portion (the red line) of zone IV, which was retained for the postoperative monitor.

noplasty. An ellipse lower abdominal flap measuring $37 \mathrm{~cm} \times 13$ $\mathrm{cm}$ was designed. The width of the flap and excision of the periumbilical skin were designed according to the distance from the superior border to the IMF on the meridian of the contralateral breast. A hand-held Doppler probe was used to check the location of the perforators on both sides.

The procedure was carried out with a two-team approach. Harvest of the DIEP flap was performed by the first team. Flap elevation began from the left side to expose the preferred perforators as planned, routine dissection of the left pedicle vessels was carried out, with one perforator artery with a visual pulse and its vena comitans on the right side also being identified as a backup. Indocyanine green (ICG) fluorescence imaging was employed to evaluate the perfusion of the flap while clamping the bilateral candidate pedicle vessels, and this showed dominant perfusion from the right side so the flap was switched to a bipedicle pattern (Fig. 4). 

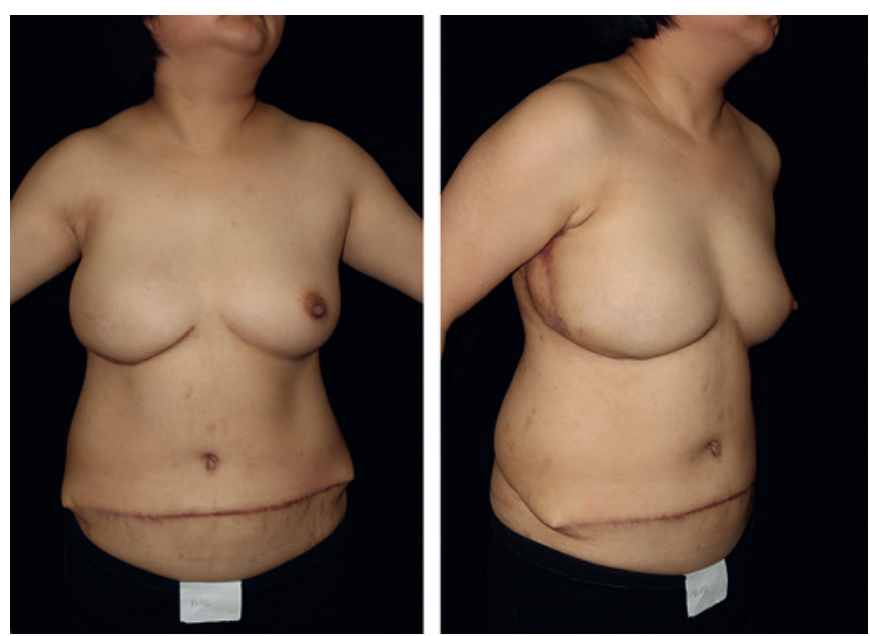

Fig. 6. The appearance of bilateral breasts at the 6-month followup. Left: frontal view. Right: oblique view.

The second team prepared the recipient site. The incision was made along the lateral border of the reconstructed breast to remove the expander and capsule as well as to explore the thoracodorsal vessels. During this process, the thoracodorsal artery was found to be of an inadequate caliber $(0.8 \mathrm{~mm}$ in diameter $)$ and was thus abandoned, while the vein with a caliber of $1.0 \mathrm{~mm}$ was retained and skeletonized for anastomoses. The incision was extended to the IMF, and the overlying skin was lifted to allow dissection of the internal mammary vessels. Both the artery and veins were of an adequate caliber and suitable for anastomoses. The DIEP flap was then rotated $180^{\circ}$ and was inset with temporary stitches. Microsurgical end-to-end anastomoses between the internal mammary vessels and the left deep inferior epigastric vessels, thoracodorsal vein, and right deep inferior epigastric vein were performed, respectively. ICG imaging showed satisfactory perfusion of zone III and the medial part of zone IV; the distal portion, measuring $3 \mathrm{~cm}$ in width, had a relatively weak signal (Fig. 5). According to this outcome, this part of the flap was retained as a skin paddle for postoperative flap monitoring and its resection in the revision procedure was planned. The rest of the flap was de-epithelialized and buried into the skin pocket to reconstruct the breast mound.

A small area of fat necrosis was encountered in the distal portion of the flap and was healed after minor debridement. The rest of the postoperative course was uneventful. The patient was discharged on day 7 . Satisfactory breast symmetry was achieved with adequate projection and a natural contour (Fig. 6).

\section{Discussion}

Thoracic deformities of Poland syndrome present with a spectrum of complex congenital skeletal and softtissue defects, affecting the ipsilateral skin, subcutaneous tissue, breast parenchyma, pectoral muscles, and underlying rib cage. In female patients, a large quantity of skin, as well as subcutaneous soft tissue, is required for the simultaneous restoration of the chest-wall depression and the reconstruction of the breast mound, offering a surgi- cal challenge depending on the severity and combination of the anatomical anomalies. In addition, the symmetry of the breast and nipple-areolar complex adds to the complexity of the situation.

Prosthetic implant reconstruction has been the mainstay of treatment; however, because of the poor coverage resulting from the underdeveloped pectoralis major muscle and the lack of skin and subcutaneous tissue in the affected chest area, there is frequent occurrence of longterm complications such as implant exposure, slippage, contour irregularities, capsular contracture, and discomfort, which lead to a high revision rate [5]. Autologous tissue reconstruction yields better results, in terms of its longevity and natural appearance. Still, the proper skin pocket and breast volume are hard to achieve by reported methods. The LD flap and transverse gracilis musculocutaneous flap carry limited skin as well as soft tissue, and the $\mathrm{LD}$ muscle is absent or there is congenital hypoplasia in some patients [17], making it impossible to reconstruct a large breast with adequate projection when these are used independently [18]. The TRAM flap provides a greater amount of tissue, but the major drawbacks here include donor-site morbidity and the loss of abdominal strength, which can be inconvenient for active patients $[19,20]$. Omental flaps provide adipose tissue as an ideal supply of autologous filling material but are only suitable for patients with sufficient skin in the affected area, meaning that implants often have to be used [10]. Fat grafting is an autologous option without the need for microsurgery, but it is suitable only in mild cases with minor asymmetry $[8,9]$.

We performed a literature search using PubMed and Google Scholar and the keywords "Poland syndrome," "breast reconstruction," "chest-wall reconstruction," and "perforator flap." All papers in the English language were reviewed as well as the reference list of each publication. Articles examining the use of other reconstructive methods such as silicone breast/chest-wall prostheses, musculocutaneous flaps, and fat injections, or that focused on other congenital breast deformities were excluded. Reports of male cases were noted and referenced but not included in the summary. Flap donor sites, recipient vessels, previous surgeries, postoperative complications, and revision surgery of all cases were summarized. Repeated cases or one with incomplete clinical information were ruled out artificially.

Overall, 7 articles describing a total of 15 cases using free perforator flaps met the inclusion criteria and were examined in depth. Primary and secondary reconstruction cases were summarized separately (Table 1). Flap donor sites included IGAP $(n=3)$, SGAP $(n=5)$, SIEA $(n=$ 2 ), anterior lateral thigh (ALT, $n=1$ ), profunda artery perforator (PAP, $n=1)$, and DIEP $(n=4)$. There was no combination use of silicone prosthesis in any of these cas- 
Table 1. Clinical reports of free perforator flaps used for breast and chest-wall reconstruction in Poland syndrome

\begin{tabular}{|c|c|c|c|c|c|c|c|}
\hline $\begin{array}{l}\text { First author } \\
\text { [Ref.] }\end{array}$ & Year & $\begin{array}{l}\text { Perforator } \\
\text { flaps, } n \\
\text { (case No.) }\end{array}$ & $\begin{array}{l}\text { Previous } \\
\text { surgery }\end{array}$ & $\begin{array}{l}\text { Vascular } \\
\text { territory }\end{array}$ & Recipient vessels & Complications & Revision \\
\hline \multirow[t]{2}{*}{$\begin{array}{l}\text { Longaker } \\
{[15]}\end{array}$} & 1997 & $1(1)$ & none & IGAP & thoracodorsal vessels & none & $\begin{array}{l}\text { contralateral breast }+ \\
\text { buttock adjustment }\end{array}$ \\
\hline & & $2(1)$ & expander & $\begin{array}{l}\text { bilateral } \\
\text { SGAP }\end{array}$ & $\begin{array}{l}\text { (1) thoracodorsal vessels } \\
\text { (2) thoracodorsal artery + } \\
\text { subscapular vein; reop. to } \\
\text { cephalic vein }\end{array}$ & $\begin{array}{l}\text { (1) flap failure } \\
\text { (2) venous congestion }\end{array}$ & none \\
\hline Liao [12] & 2005 & $1(1)$ & none & DIEP & internal mammary vessels & none & none \\
\hline \multirow[t]{6}{*}{$\begin{array}{l}\text { Gautam } \\
{[14]}\end{array}$} & 2007 & $3(3)$ & none & SGAP & internal mammary vessels & none & $\begin{array}{l}\text { contralateral breast } \\
\text { adjustment }(n=1)\end{array}$ \\
\hline & & $1(1)$ & implant & SGAP & internal mammary vessels & hematoma & none \\
\hline & & $\overline{1(1)}$ & implant & SIEA & internal mammary vessels & donor-site seroma & none \\
\hline & & $1(1)$ & implant & IGAP & internal mammary vessels & $\begin{array}{l}\text { venous congestion } \\
\text { hematoma }\end{array}$ & none \\
\hline & & $1(1)$ & implant & DIEP & internal mammary vessels & fat necrosis & none \\
\hline & & $1(1)$ & $\begin{array}{l}\text { implant, } \\
\text { LD flap }\end{array}$ & IGAP & internal mammary vessels & none & none \\
\hline $\begin{array}{l}\text { Blechman } \\
{[23]}\end{array}$ & 2013 & $2(1)$ & implant & $\begin{array}{l}\text { bilateral } \\
\text { PAP }\end{array}$ & $\begin{array}{l}\text { (1) internal mammary vessels } \\
\text { retrograde } \\
\text { (2) internal mammary vessels } \\
\text { anterograde }\end{array}$ & none & none \\
\hline Dolas [24] & 2014 & $1(1)$ & none & ALT & thoracodorsal vessels & none & none \\
\hline Masia [13] & 2015 & $2(1)$ & none & $\begin{array}{l}\text { (1) DIEP } \\
\text { (2) SIEA }\end{array}$ & $\begin{array}{l}\text { (1) thoracodorsal vessels } \\
\text { (2) internal mammary vessels }\end{array}$ & none & none \\
\hline $\begin{array}{l}\text { Dionyssiou } \\
{[28]}\end{array}$ & 2015 & $1(1)$ & implant & DIEP & thoracodorsal vessels & none & none \\
\hline
\end{tabular}

IGAP, inferior gluteal artery perforator; SGAP, superior gluteal artery perforator; DIEP, deep inferior epigastric perforator; SIEA, superficial inferior epigastric artery; PAP, profunda artery perforator; ALT, anterior lateral thigh; LD, latissimus dorsi.

es. Seven of the 15 cases were primary reconstructions, for which no major complications were reported. The other 8 cases were revision surgeries due to the failure of previous implants $(n=7)$ and expanders $(n=1)$; complications included venous congestion $(n=2)$, hematoma $(n=2)$, seroma $(n=1)$, fat necrosis $(n=1)$, and flap loss $(n=1)$. Satisfactory correction was reported in all the cases, with adjustment of the contralateral breast being performed to optimize symmetry in 2 cases.

Perforator flaps are known to make use of a patient's excessive skin and adipose tissue for reconstruction while minimizing donor-site morbidity [21]. In 1997, Longaker et al. [15] first reported a case using IGAP flaps for Poland syndrome breast reconstruction. Since then, sporadic discussion regarding different flap donor sites has demonstrated the capability of perforator flaps to adapt to various settings of breast and chest wall deformity of Poland syndrome-associated anomalies. As the most popular perforator flap for postmastectomy breast reconstruction, the DIEP flap is recommended for treating Poland syndrome also because of its ample volume and significantly longer pedicles which allow for more freedom in tissue-tailoring and flap insetting. SGAP [22] and ALT $[24,25]$ flaps served as alternatives in cases of inadequate abdominal tissue or previous scars. Gautam et al. [14] reported the largest series of revision surgeries using perforator flaps for previous implant or flap failure that achieved satisfying long-time results, despite the greater occurrence of short-term complications than with primary reconstructions including hematoma, seroma, and fat necrosis.

Here, we report an innovative two-stage strategy for breast reconstruction in Poland syndrome. It is the first reported case, to the best of our knowledge, to use a staged tissue expander/DIEP flap to achieve pleasing breast and chest-wall symmetry. The tissue expansion technique was employed as the first stage to create an ideal skin envelope large enough to accommodate the entire DIEP flap, thus avoiding the reconstructed breast having a patch-like appearance. The expanding process also provided the op- 
portunity to define the breast border and predict the tissue volume needed for the second stage. Intraoperatively, ICG imaging and additional anastomoses of the contralateral pedicle vessels were performed to facilitate flap perfusion and maximize the volume of well-vascularized tissue. The DIEP flap was rotated through $180^{\circ}$ and a wedge of periumbilical skin was excised to gather tissue at the lower pole to obtain a ptosis breast appearance. Zones I and II of the flap, with better vascularization, were placed medially, making sure to preserve enough tissue to fill the upper medial quadrant of the breast. Zone IV of the flap was folded cranially to fill the empty infraclavicular and axillary area. The expanded skin flap was undermined on lateral and cranial borders of the footprint, so the edge of the flap could be inserted to create a smooth transition.

Compared with straightforward IMF incision, the endoscopic axillary approach we chose for expander insertion remained distant from the expanding area, resulting in minor restriction for the tissue to expand, reducing the probability of expander exposure, and limiting scar migration during the expansion process. In the second stage of flap transfer, this incision could be extended along the IMF to expose the thoracodorsal and internal mammary vessels for anastomoses, while at the same time kept concealed at the breast border.

The main disadvantages of this procedure include an extended operative time and relatively high costs for the patient. Microsurgical expertise is required, and complications including partial necrosis, flap failure, and fat necrosis must be discussed with the patient. The prolonged treatment duration and multiple operative room visits should be noted as well. Simultaneous reconstruction of the breast and anterior axillary fold can be quite difficult due to the limitation of flap insetting. Masia et al. [13] reported a reconstructive case using a split flap, in which the hemi-DIEP flap was transferred to correct the chest deformity and the contralateral SIEA flap was used for the breast reconstruction. This provided sufficient tissue and allowed for better reshaping at the same time.

Possible vascular anomalies related to thoracic deformities can complicate the situation and make intraoperative assessment mandatory [26]. In our case, the vessels from the ipsilateral subclavian system including the thoracodorsal artery, lateral thoracic artery, and thoracoacromial artery were each explored but yielded either an undersized caliber or weak pulsing. We assume this condition resulted from the vascular anomaly associated with Poland syndrome. The same phenomenon was mentioned by Longaker et al. [15], in whose series one free flap loss was assumed to have been due to anomalous subclavian venous drainage. Bouvet et al. [27] found a marked decrease in arterial blood flow velocity on the affected side, suggesting hypoplasia in the subclavian artery. There has been little discussion and research regarding this issue; we believe this is essential for the future practice of using perforator flaps for Poland syndrome. On the other hand, the anatomy of the internal mammary vessels was of a high stability, according to the published articles and our own experience. We recommend, whenever possible, careful exploration of these vessels and the preservation of the contralateral DIEPs as the "lifeboat" before a full assessment.

Utilizing perforator flaps for Poland syndrome breast reconstruction provides reliable cosmetically satisfactory results and avoids implant-related complications. Thorough pre- and intraoperative assessments are vital for effective application. Larger-scale clinical studies are needed for the further discussion and evaluation of this method.

\section{Statement of Ethics}

All procedures performed in studies involving human participants were in accordance with the ethical standards of the institutional research committee and the 1964 Helsinki Declaration and its later amendments or comparable ethical standards. The participant gave her informed consent in writing prior to her inclusion in the study.

\section{Disclosure Statement}

The authors have no conflicts of interest to declare.

\section{Funding Sources}

This study was supported by Capital's Funds for Health Improvement and Research (No. 2016-2-4041), the PUMC Youth Fund, and Fundamental Research Funds for the Central Universities (No. 3332015156) (to C.L.).

\section{Author Contributions}

B.X. was the major contributor to writing the manuscript. T.L. acquired the clinical data and performed the literature search. C.L. was the senior surgeon who performed the operation. All authors read and approved the final manuscript.

References

1 Poland A. Deficiency of the pectoral muscles. Guys Hosp Rep. 1841;6:191-3.

2 da Silva Freitas R, Dall'Oglio Tolazzi AR, Martins VD, Knop BA, Graf RM, de Oliveira e Cruz GA. de Oliveira e Cruz GA. Poland's Syndrome: Different Clinical Presentations and Surgical Reconstructions in 18 Cases. Aesthetic Plast Surg. 2006 Nov;31(2):140-6.

3 Majdak-Paredes EJ, Shafighi M, Fatah F. Integrated algorithm for reconstruction of complex forms of Poland syndrome: 20-year outcomes. J Plast Reconstr Aesthet Surg. 2015 Oct;68(10):1386-94. 
4 Pereira LH, Sabatovich O, Santana KP, Picanço R, Sterodimas A. Surgical correction of Poland's syndrome in males - a purposely designed implant. J Plast Reconstr Aesthet Surg. 2008;61(4):393-9.

5 Seyfer AE, Fox JP, Hamilton CG. Poland syndrome: evaluation and treatment of the chest wall in 63 patients. Plast Reconstr Surg. 2010 Sep;126(3):902-11.

6 Hokin JA, Silfverskiold KL. Breast reconstruction without an implant: results and complications using an extended latissimus dorsi flap. Plast Reconstr Surg. 1987 Jan; 79(1):58-66.

7 Tvrdek M, Kletenský J, Svoboda S. Aplasia of the breast-reconstruction using a free tram flap. Acta Chir Plast. 2001;43(2):39-41.

8 Delay E, Sinna R, Chekaroua K, Delaporte T, Garson S, Toussoun G. Lipomodeling of Poland's syndrome: a new treatment of the thoracic deformity. Aesthetic Plast Surg. 2010 Apr;34(2):218-25.

9 Pinsolle V, Chichery A, Grolleau JL, Chavoin JP. Autologous fat injection in Poland's syndrome. J Plast Reconstr Aesthet Surg. 2008 Jul;61(7):784-91.

10 dos Santos Costa S, Blotta RM, Mariano MB, Meurer L, Edelweiss MI. Aesthetic improvements in Poland's syndrome treatment with omentum flap. Aesthetic Plast Surg. 2010 Oct; 34(5):634-9.

11 Hamdi M, Weiler-Mithoff EM, Webster MH. Deep inferior epigastric perforator flap in breast reconstruction: experience with the first 50 flaps. Plast Reconstr Surg. 1999 Jan; 103(1):86-95.

12 Liao HT, Cheng MH, Ulusal BG, Wei FC. Deep inferior epigastric perforator flap for successful simultaneous breast and chest wall reconstruction in a Poland anomaly patient. Ann Plast Surg. 2005 Oct;55(4):422-6.
13 Masia J, Pons G, Loschi P, Sanchez Porro-Gil L, Nardulli ML, Olivares L. Autologous reconstruction of a complex form of Poland syndrome using 2 abdominal perforator free flaps. Ann Plast Surg. 2015 May;74(5):580-3.

14 Gautam AK, Allen RJ Jr, LoTempio MM, Mountcastle TS, Levine JL, Allen RJ, et al. Congenital breast deformity reconstruction using perforator flaps. Ann Plast Surg. 2007 Apr;58(4):353-8.

15 Longaker MT, Glat PM, Colen LB, Siebert JW. Reconstruction of breast asymmetry in Poland's chest-wall deformity using microvascular free flaps. Plast Reconstr Surg. 1997 Feb; 99(2):429-36

16 Liu C, Luan J, Mu L, Ji K. The role of threedimensional scanning technique in evaluation of breast asymmetry in breast augmentation: a 100-case study. Plast Reconstr Surg. 2010 Dec;126(6):2125-32.

17 Cochran JH Jr, Pauly TJ, Edstrom LE, Dibbell DG. Hypoplasia of the latissimus dorsi muscle complicating breast reconstruction in Poland's syndrome. Ann Plast Surg. 1981 May; 6(5):402-4.

18 Huemer GM, Puelzl P, Schoeller T. Breast and chest wall reconstruction with the transverse musculocutaneous gracilis flap in Poland syndrome. Plast Reconstr Surg. 2012 Oct;130(4): 779-83.

19 Kaplan JL, Allen RJ. Cost-based comparison between perforator flaps and TRAM flaps for breast reconstruction. Plast Reconstr Surg. 2000 Mar;105(3):943-8.

20 Blondeel N, Vanderstraeten GG, Monstrey SJ, Van Landuyt K, Tonnard P, Lysens R, et al. The donor site morbidity of free DIEP flaps and free TRAM flaps for breast reconstruction. Br J Plast Surg. 1997 Jul;50(5):322-30.
21 Koshima I, Soeda S. Inferior epigastric artery skin flaps without rectus abdominis muscle. Br J Plast Surg. 1989 Nov;42(6):645-8.

22 Guerra AB, Metzinger SE, Bidros RS, Gill PS, Dupin CL, Allen RJ. Breast reconstruction with gluteal artery perforator (GAP) flaps: a critical analysis of 142 cases. Ann Plast Surg. 2004 Feb;52(2):118-25.

23 Blechman KM, Broer PN, Tanna N, Ireton JE, Ahn CY, Allen RJ. Stacked profunda artery perforator flaps for unilateral breast reconstruction: a case report. J Reconstr Microsurg. 2013 Nov;29(9):631-4.

24 Dolas SC, Poovamma CU, Prema M, Khandelwal R, Pais AV, Kaul A. Poland's syndrome: a case report with review of literature regarding management. Breast Dis. 2014; 34(3):121-5.

25 Gravvanis A, Lo S, Shirley R. Aesthetic restoration of Poland's syndrome in a male patient using free anterolateral thigh perforator flap as autologous filler. Microsurgery. 2009; 29(6):490-4.

26 Bavinck JN, Weaver DD, Opitz JM, Reynolds JF. Subclavian artery supply disruption sequence: hypothesis of a vascular etiology for Poland, Klippel-Feil, and Möbius anomalies. Am J Med Genet. 1986 Apr;23(4):903-18.

27 Bouvet JP, Leveque D, Bernetieres F, Gros JJ. Vascular origin of Poland syndrome? A comparative rheographic study of the vascularisation of the arms in eight patients. Eur J Pediatr. 1978 May;128(1):17-26.

28 Dionyssiou D, Demiri E, Batsis G, Pavlidis L. Revision breast and chest wall reconstruction in Poland and pectus excavatum following implant complication using free deep inferior epigastric perforator flap. Indian J Plast Surg. 2015;48(1):85-8. 